\title{
More Than Just Adolescence: Differences in Fatigue Between Youth With Cerebral Palsy and Typically Developing Peers
}

\author{
Laura K. Brunton, PT, PhD ${ }^{1}$, Elizabeth G. Condliffe, MD, PhD ${ }^{2}$, Gregor Kuntze, $\mathrm{PhD}^{3}$, \\ Leticia Janzen, $\mathrm{MSc}^{3}$, Shane Esau, $\mathrm{MK}^{3}$, Clodagh $\mathrm{M}$. Toomey, $\mathrm{PhD}^{4}$, Carolyn A. Emery, $\mathrm{PhD}^{3}$ \\ ${ }^{1}$ School of Physical Therapy, Western University, London, Ontario; \\ ${ }^{2}$ Cumming School of Medicine, University of Calgary, Calgary, Alberta; \\ ${ }^{3}$ Faculty of Kinesiology, University of Calgary, Calgary, Alberta, Canada; \\ ${ }^{4}$ School of Allied Health, University of Limerick, Limerick, Ireland
}

Objective To quantify differences in fatigue and disordered sleep between adolescents with cerebral palsy (CP) and their typically developing peers. A secondary aim was to investigate the association between fatigue and disordered sleep in adolescents with CP.

Methods A convenience sample of 36 youth with CP aged 10-18 years was matched for age and sex with 36 typically developing peers. The Fatigue Impact and Severity Self-Assessment (FISSA), the Patient-Reported Outcome Measurement Information System (PROMIS) fatigue profile, and the Sleep Disturbance Scale for Children (SDSC) were collected.

Results Higher fatigue was reported in participants with CP than in their typically developing peers based on the FISSA total score (mean paired difference=19.06; 99\% confidence interval [CI], 6.06-32.1), the FISSA impact subscale (mean paired difference $=11.19 ; 99 \% \mathrm{CI}, 3.96-18.4$ ), and the FISSA Management and Activity Modification subscale (mean paired difference $=7.86 ; 99 \% \mathrm{CI}, 1.1-14.6$ ). There were no differences between groups in the PROMIS fatigue profile (mean paired difference $=1.63$; 99\% CI, -1.57-4.83) or the SDSC total score (mean paired difference $=2.71 ; 99 \% \mathrm{CI},-2.93-8.35)$.

Conclusion Youth with CP experienced significantly more fatigue than their peers as assessed by a comprehensive measure that considered both general and diagnosis-specific concerns. Sleep did not differ between youth with $\mathrm{CP}$ and their typically developing peers. These findings underscore the need to consider the clinical management of fatigue across the lifespan of individuals with $\mathrm{CP}$ to prevent the associated deterioration of functional abilities.

Keywords Cerebral palsy, Fatigue, Sleep, Adolescent

Received December 8, 2020; Revised February 17, 2021; Accepted March 15, 2021; Published online June 14, 2021

Corresponding author: Laura K. Brunton

School of Physical Therapy, Western University, 1588 Elborn College, London, Ontario N6G1H1, Canada. Tel: +1-519-661-2111 (ext. 81501), Fax: +1519-661-3866, E-mail: lbrunto3@uwo.ca

ORCID: Laura K. Brunton (https://orcid.org/0000-0002-0890-7040); Elizabeth G. Condliffe (https://orcid.org/0000-0002-0178-616X); Gregor Kuntze (https://orcid.org/0000-0001-6674-7981); Leticia Janzen (https://orcid.org/0000-0002-8582-5209); Shane Esau (https://orcid.org/0000-0001-56141011); Clodagh M. Toomey (https://orcid.org/0000-0002-9373-100X); Carolyn A. Emery (https://orcid.org/0000-0002-9499-6691).

(c) This is an open-access article distributed under the terms of the Creative Commons Attribution Non-Commercial License (http://creativecommons.org/ licenses/by-nc/4.0) which permits unrestricted noncommercial use, distribution, and reproduction in any medium, provided the original work is properly cited. Copyright $\odot 2021$ by Korean Academy of Rehabilitation Medicine 


\section{INTRODUCTION}

Fatigue is defined as a "self-recognized state in which an individual experiences an overwhelming and sustained sense of exhaustion and decreased capacity for physical and mental work, that is not relieved by rest" [1]. Adolescents are particularly susceptible to fatigue because of their unique developmental needs and sleep habits. Fatigue affects an estimated $40 \%-60 \%$ of all adolescents [2]. Fatigue is also common in individuals with cerebral palsy (CP), with up to $50 \%$ of adolescents and adults with CP experiencing fatigue that impacts their quality of life and daily activities [3]. Between $10 \%$ and $28 \%$ of individuals with CP report daily fatigue [3]. Depending on their level of functional ability, $70 \%-92 \%$ of adolescents with $\mathrm{CP}$ report feeling fatigued for at least one-quarter of their average day, and between $53 \%-78 \%$ report experiencing fatigue on at least 3 days of the week [3]. While youth with CP experience a significant amount of fatigue, $22 \%$ of typically developing adolescents also report experiencing fatigue most days of the week [2]. The prevalence of selfreported fatigue is similar between adolescents with and without $\mathrm{CP}$; however, different fatigue scales have been used and, until recently, no measure had been specifically validated for use with adolescents with CP [4]. Thus, the differences in fatigue between youth with $\mathrm{CP}$ and typically developing peers are currently unknown. It is important to determine whether the fatigue experienced by youth with CP differs from that experienced because of adolescence and the unique challenges posed by this stage of development.

Sleep further compounds fatigue. Adolescents require longer sleep times, experience more daytime sleepiness, and have large shifts in circadian rhythms that often result in later bed and rise times [5]. In addition, adolescents often have poor sleep habits that may be related to school schedules, homework, extended screen time, and social commitments. However, sleep disorders are more frequent in children with $\mathrm{CP}$ than in their typically developing peers [6-8]. An estimated $20 \%$ of children with CP have abnormal total sleep scores and more than $40 \%$ of children with $\mathrm{CP}$ have at least one clinically significant sleep disorder $[6,9]$. The high-prevalence of disordered sleep has been hypothesized to be partially due to muscle spasms and other forms of musculoskeletal pain related to primary motor impairment in CP. However, there is limited evidence regarding the relationship between physical impairment and sleep. Previous studies have demonstrated an association between fatigue and the characteristics of $\mathrm{CP}$ and its complications $[4,6,10]$, and that management of spasticity and pain improves sleep $[11,12]$. However, the relationship between fatigue and sleep disturbances in young people with $\mathrm{CP}$ has not been studied.

The primary objective of this study was to quantify differences in fatigue and disordered sleep between adolescents with CP and their age- and sex-matched typically developing peers. The secondary objective was to investigate the association between fatigue and disordered sleep in adolescents with CP.

\section{MATERIALS AND METHODS}

\section{Design}

This study was a sub-study of a matched-pair cohort study design comparing physical activity, gait parameters, fatigue, body composition, and sleep of individuals with CP to those of their typically developing peers [13]. This study was approved by the Conjoint Health Research Ethics Board at the University of Calgary (Ethics ID. REB15-3126) and informed consent and assent was obtained from all parents and children prior to data collection.

\section{Participants}

A convenience sample of youth with $\mathrm{CP}$ was recruited and matched with typically developing peers for age (within 18 months) and sex. The participants were recruited from the Neuromotor Clinic (CP) and the Healthy Infants and Children's Clinical Research Program (typically developing) at the Alberta Children's Hospital in Calgary, Alberta, Canada, between 2016 and 2020. Additional typically developing participants were recruited using a snowball sampling approach targeting siblings and friends of children with CP in the study and by word of mouth. Care was taken to ensure that all matches were made to participants that were not known to each other to reduce the associated influences that may exist within family or social environments. The inclusion criteria for participants in both groups included adolescents aged 10-18 years and the ability to complete self-report questionnaires in English. The additional inclusion criteria for 
participants with CP were ongoing clinical diagnosis of $\mathrm{CP}$ with the presence of spasticity (spastic or mixed tone) and Gross Motor Function Classification System (GMFCS) levels I-III. The exclusion criteria for both groups were the inability to follow instructions or complete self-report questionnaires in English, a current or past diagnosis of an unrelated neurological condition other than $\mathrm{CP}$, a musculoskeletal injury within 3 months before testing from which they had not fully recovered, and any current medical condition that prevented their participation in functional testing. The additional exclusion criteria for the group with CP included botulinum toxin injections within 6 months before the testing date, a surgical procedure from which they had not fully recovered within 1 year before the testing date, and uncontrolled epilepsy.

\section{Procedures}

The participants completed a demographic questionnaire and three self-report questionnaires: (1) the Fatigue Impact and Severity Self-Assessment (FISSA), (2) the Patient-Reported Outcome Measurement Information System (PROMIS) 49-item version pediatric profile, and (3) the Sleep Disturbance Scale for Children (SDSC). The FISSA was created to assist in identifying individuals experiencing fatigue related to $\mathrm{CP}$ and to promote discussions between clinicians and their patients about fatigue and possible management strategies [4]. The FISSA is a 37 -item questionnaire, the first 31 questions of which are generally scored using a 5-point Likert scale ranging from 1 (completely disagree) to 5 (completely agree). However, questions related to the severity of fatigue have a variety of scaling options related to individual questions, as appropriate [4]. The remaining six questions ask participants about their diurnal patterns of fatigue as well as open-ended questions about managing fatigue. The overall total fatigue score was calculated by summing the responses to the first 31 questions and expressing the level of fatigue experienced in terms of both impact and severity (range, 31-157 scores) [4]. The FISSA contains two subscales: the Impact Scale (subscale score calculated by summing the responses to items \#1-17; range 17-87 scores) and the Management and Activity Modification scale (subscale score calculated by summing the responses to items \#18-31; range, 14-70 scores) [4]. The FISSA has shown moderate test-retest reliability, with an intraclass correlation coefficient of 0.75 (95\% confidence interval [CI], 0.54-0.87) and high internal consistency, with a Cronbach's alpha of 0.95 [4]. The content validity of the questionnaire was ensured during the development stages of the FISSA, and known-group validity was established by assessing fatigue in the high and low pain groups and by GMFCS levels [4].

The PROMIS-49 is a general health status measure with seven domains (physical function, mobility, anxiety, depressive symptoms, fatigue, peer relationships, pain interference, and pain intensity). The present study used the PROMIS-49 fatigue item bank (eight items; range, 0-32 scores), with each item scored on a frequency-based 5 -point Likert Scale $(0=$ never, $1=$ almost never, $2=$ sometimes, $3=$ often, $4=$ almost always). The PROMIS fatigue profile has been extensively tested and found to be psychometrically sound for children with multiple chronic conditions [14].

Parents, with the help of the youth, completed the SDSC [15]. The SDSC was originally validated on a large sample of healthy children and published norms are available [15]. The SDSC contains 26 items and measures the occurrence of sleep disorders in the past 6 months, rated on a 5-point Likert-type scale [15]. Scores can be summed to provide a total sleep score (range, 26-130 scores) [15]. This scale was used in the present study due to its comprehensive validation, high level of internal consistency, test-retest reliability, and availability of normative data corresponding to the age group investigated in the present study.

\section{Statistical analysis}

Descriptive statistics, including mean \pm standard deviation and frequencies (proportion), were used to summarize all baseline characteristics of the participants. The normality of the data was assessed using the ShapiroWilk test. Differences between participants with CP and typically developing youth were examined using pairedsample t-tests with Bonferroni correction to account for multiple comparisons $(\mathrm{p}<0.01 ; 0.05 / 5)$. Differences between pairs were summarized using mean paired differences and 99\% CIs. Separate hierarchical linear regression analyses were used to determine the associations between the PROMIS fatigue profile and the FISSA total score, the PROMIS fatigue profile and the SDSC total score, and FISSA and the SDSC total scores after controlling for clustering by pair. 


\section{RESULTS}

A total of 36 age-and-sex matched pairs were enrolled in the study (see baseline characteristics, Table 1). Higher levels of fatigue were reported in participants with CP than in their typically developing peers based on the FISSA total score (mean paired difference $=19.06$; $99 \%$ CI, 6.06-32.1), the FISSA impact subscale (mean paired difference $=11.19 ; 99 \% \mathrm{CI}, 3.96-18.4$ ), and the FISSA Management and Activity Modification subscale (mean paired difference $=7.86$; 99\% CI, 1.1-14.6) (Table 2). There were no differences in the PROMIS fatigue profile (mean paired difference $=1.63 ; 99 \% \mathrm{CI},-1.57-4.83]$ ) or the SDSC

Table 1. Participant characteristics

\begin{tabular}{lcc}
\hline \multicolumn{1}{|c}{ Characteristic } & $\begin{array}{c}\text { Cerebral } \\
\text { palsy }\end{array}$ & $\begin{array}{c}\text { Typically } \\
\text { developing }\end{array}$ \\
\hline Sex & $24(67)$ & $24(67)$ \\
\hline Male & $12(33)$ & $12(33)$ \\
\hline Female & $14.14 \pm 2.37$ & $14.14 \pm 2.55$ \\
\hline Age (yr) & $19(53)$ & - \\
\hline GMFCS level & $13(36)$ & - \\
\hline I & $4(11)$ & - \\
\hline II & $18(50)$ & - \\
\hline III & $18(50)$ & - \\
\hline Topographical distribution of involvement & \\
\hline Unilateral & & \\
\hline Bilateral &
\end{tabular}

Values are presented as number of participants (\%) or mean \pm standard deviation.

GMFCS, Gross Motor Function Classification System. total score (mean paired difference $=2.71 ; 99 \% \mathrm{CI},-2.93$ 8.35) between groups.

After accounting for paired clusters, an association was observed between the PROMIS fatigue profile score and the FISSA total score $\left(\beta=0.550, R^{2}\right.$ change $\left.=0.298 ; p<0.001\right)$ (Fig. 1A). Significant relationships were also observed between the FISSA total score and the SDSC total score $\left(\beta=0.297, R^{2}\right.$ change $\left.=0.086 ; p=0.01\right)$ and between the PROMIS fatigue profile and the SDSC total score $(\beta=0.420$, $\mathrm{R}^{2}$ change $=0.176 ; \mathrm{p}<0.001$ ) (Fig. $1 \mathrm{~B}, 1 \mathrm{C}$ ). Overall, fatigue accounted for $8 \%$ to $18 \%$ of the variance in the total sleep score.

\section{DISCUSSION}

This study is the first to evaluate the differences in fatigue between youth with CP and their typically developing peers. Using the FISSA, we demonstrated that youth with CP experienced higher total fatigue and a higher impact and severity of fatigue compared to their peers without a disability. Additionally, we observed significant differences between groups in the Management and Activity Modification subscale of the FISSA, despite the lack of evidence-based fatigue management interventions as part of routine practice for youth with $\mathrm{CP}$. This finding indicates youth with CP may try to self-manage the impacts of fatigue in their day-to-day life to a greater extent than their peers.

The PROMIS fatigue profile did not differ significantly between the groups, indicating that both diagnosis-specific and general factors related to adolescence contribute to the fatigue experienced by youth with CP. These

Table 2. Group means and matched pair differences

\begin{tabular}{lcccc}
\hline & Cerebral palsy & Typically developing & $\begin{array}{c}\text { Mean difference } \\
(\mathbf{9 9 \%} \text { CI) }\end{array}$ & p-value \\
\hline FISSA total score & $75.81 \pm 25.92$ & $56.75 \pm 19.54$ & $19.06(6.06-32.10)$ & $0.001^{* * *}$ \\
FISSA impact subscale score & $38.72 \pm 14.66$ & $27.52 \pm 9.43$ & $11.19(3.96-18.40)$ & $<0.001^{* * *}$ \\
$\begin{array}{l}\text { FISSA management and activity } \\
\text { modification subscale score }\end{array}$ & $37.08 \pm 12.45$ & $29.22 \pm 11.38$ & $7.86(1.10-14.60)$ & $0.005^{* *}$ \\
PROMIS fatigue profile & & & & \\
SDSC total score & $6.40 \pm 5.18$ & $4.77 \pm 5.07$ & $1.63(-1.57-4.83)$ & 0.199 \\
\hline
\end{tabular}

Values are presented as mean \pm standard deviation.

FISSA, Fatigue Impact and Severity Self-Assessment; PROMIS, Patient-Reported Outcome Measurement Information System; SDSC, Sleep Disturbance Scale for Children; CI, confidence interval.

${ }^{* *} \mathrm{p}<0.01,{ }^{* * *} \mathrm{p}<0.001$. 

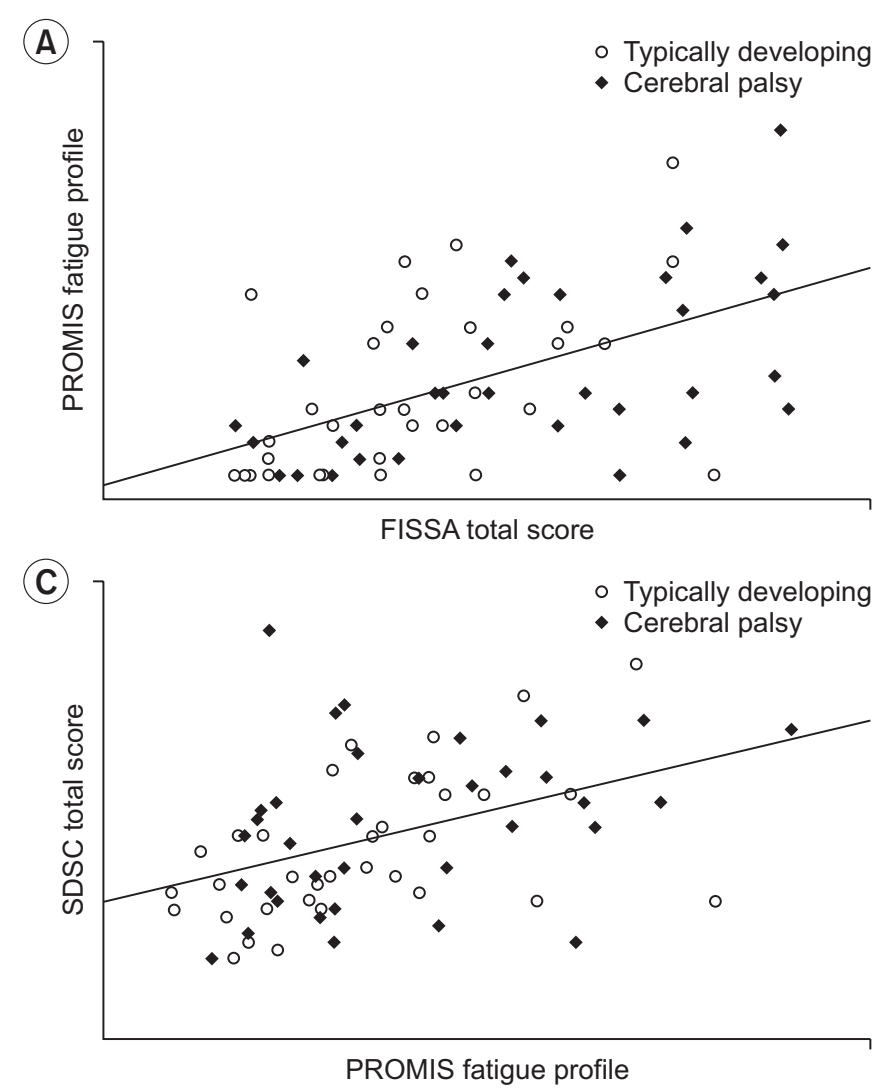

findings also underscore the need to consider the range and sensitivity of measurement tools for assessing fatigue in this population. The use of a tool created and validated to measure specific aspects of CP-related fatigue, such as the FISSA, is imperative as it deepens the understanding of fatigue, targeting specific challenges created by the diagnosis of $\mathrm{CP}$ that are beyond the capacity of general measures such as the PROMIS-49. The correlation between the PROMIS fatigue profile and the FISSA indicated that both measures assess a similar construct, fatigue. However, the PROMIS fatigue profile contains only eight items, focused on generalized experiences with fatigue, and may not be representative of the full experience, severity, and impact of fatigue and/or be sensitive enough to detect the resulting differences between groups. In contrast, the FISSA contains a wider breadth of items and incorporates multiple domains including impact, severity, and activity modification or management strategies to describe in greater detail the experience of fatigue in youth with CP. Specifically, the differences in the total FISSA fatigue score could be driven by the larger impact of fatigue on daily activities and increased severity of fatigue for youth with CP, as evidenced by the higher sub-



Fig. 1. Relationships between fatigue measures and fatigue and sleep in youth with cerebral palsy and typically developing peers using hierarchical linear regression controlled for cluster pairs: (A) PROMIS fatigue profile and FISSA total score, (B) SDSC total score and FISSA total score, and (C) SDSC total score and PROMIS fatigue profile. FISSA, Fatigue Impact and Severity Self-Assessment; PROMIS, Patient-Reported Outcome Measurement Information System; SDSC, Sleep Disturbance Scale for Children.

scale score, due to condition-specific functional limitations associated with tasks of daily living. Combined with the observed differences in the management of fatigue between typically developing youth and youth with $\mathrm{CP}$, typically developing youth may be able to continue with daily tasks despite their fatigue, while youth with $\mathrm{CP}$ experience the heightened impact of fatigue due to their reduced functional abilities, for which they require steps to mitigate their fatigue.

The levels of fatigue documented in typically developing youth in this study confirmed that some general aspects of adolescence contribute to the fatigue experienced by youth with CP. These factors may be amenable to interventions developed for the general population and could be a first target for clinicians and families supporting youth with $\mathrm{CP}$ experiencing fatigue, given that there are no evidence-based interventions to reduce $\mathrm{CP}$ related fatigue. Moreover, interventional studies aimed at reducing fatigue in youth with $\mathrm{CP}$ should consider a multidimensional approach to address the conditionspecific and general factors associated with life stage to adequately manage fatigue in this population. Impaired motor function likely contributes to fatigue, although 
evidence suggests that it is not the only contributing factor [16] and that the association between impairments in motor function and fatigue is not entirely linear $[3,17]$. Furthermore, reduced physical fitness associated with $\mathrm{CP}$ may contribute to the fatigue experienced; however, Bolster et al. [18] found that general fatigue was not explained by low fitness levels or the high energy demands associated with walking.

No differences in self-reported sleep were observed between youth with $\mathrm{CP}$ and their peers. In the regression analysis, regardless of the fatigue measure used, fatigue accounted for $<20 \%$ of the variance in the SDSC score. Furthermore, although a weak positive relationship was observed between fatigue and sleep disturbances in both the FISSA and PROMIS, the low proportion of variance explained by these models indicated that fatigue includes distinct factors that must be assessed and treated separately from addressing any potential sleep concerns in individuals with CP.

The limitations of this study include the small sample size and convenience sampling approach used to recruit study participants, which may have limited the generalizability of the findings. Furthermore, potential confounding variables such as age, sex, and gross motor function (GMFCS level) were not examined and the small sample size resulted in the inability to assess differences in fatigue or sleep in these potentially relevant subgroups. Including fatigue assessment as part of a future core dataset or a large registry study could help address these limitations and facilitate studies exploring mechanisms that may contribute to fatigue and fatigue differences across GMFCS levels. Finally, because of the use of self-report measures, measurement bias was possible.

In conclusion, this study was the first to assess if the fatigue experienced by youth with CP differed from that generally associated with the life stage of adolescence and to evaluate the relationship between fatigue and sleep in this population. Youth with CP aged 10-18 years experienced significantly more fatigue than their age-and sex-matched peers, as assessed by a comprehensive measure that considers both general and diagnosis-specific concerns. Sleep did not differ between youth with CP and their typically developing peers. These findings underscore the need to consider the clinical management of fatigue across the lifespan for individuals with $\mathrm{CP}$ to prevent the associated deterioration of functional abili- ties $[19,20]$ and to optimize functional capacity during a period defined by growth, maturation, and the development of self-management skills.

\section{CONFLICT OF INTEREST}

No potential conflict of interest relevant to this article was reported.

\section{ACKNOWLEDGMENTS}

This work was funded through a postdoctoral fellowship awarded by Alberta Innovates Health Solutions to Laura K. Brunton.

\section{AUTHOR CONTRIBUTION}

Conceptualization: Brunton LK, Emery CA Methodology: Emery CA, Brunton LK, Toomey CM, Kuntze G. Formal analysis: Brunton LK. Project administration: Brunton LK, Janzen L, Esau S. Writing-original draft: Brunton LK. Writing-review and editing: Brunton LK, Kuntze G, Condliffe EG, Toomey CM, Emery CA. Approval of the final manuscript: all authors.

\section{REFERENCES}

1. Carpenito-Moyet LJ. Nursing diagnosis: application to clinical practice. 11th ed. Philadelphia, PA: Lippincott Williams \& Wilkins; 2006.

2. Currie C, Hurrlemann K, Settertobulte W, Smith R, Todd J. Health and health behaviour among young people. Copenhagen, Denmark: World Health Organization Regional Office for Europe; 2000.

3. Brunton LK, Bartlett DJ. Profiles of fatigue severity and variability among adolescents and young adults with cerebral palsy. Fatigue 2017;5:5-14.

4. Brunton LK, Bartlett DJ. Construction and validation of the fatigue impact and severity self-assessment for youth and young adults with cerebral palsy. Dev Neurorehabil 2017;20:274-9.

5. Carskadon MA, Wolfson AR, Acebo C, Tzischinsky O, Seifer R. Adolescent sleep patterns, circadian timing, and sleepiness at a transition to early school days. Sleep 1998;21:871-81.

6. Newman CJ, O'Regan M, Hensey O. Sleep disorders in 
children with cerebral palsy. Dev Med Child Neurol 2006;48:564-8.

7. Hayashi M, Inoue Y, Iwakawa Y, Sasaki H. REM sleep abnormalities in severe athetoid cerebral palsy. Brain Dev 1990;12:494-7.

8. Kotagal S, Gibbons VP, Stith JA. Sleep abnormalities in patients with severe cerebral palsy. Dev Med Child Neurol 1994;36:304-11.

9. Romeo DM, Brogna C, Quintiliani M, Baranello G, Pagliano E, Casalino T, et al. Sleep disorders in children with cerebral palsy: neurodevelopmental and behavioral correlates. Sleep Med 2014;15:213-8.

10. Van Der Slot WM, Nieuwenhuijsen C, Van Den BergEmons RJ, Bergen MP, Hilberink SR, Stam HJ, et al. Chronic pain, fatigue, and depressive symptoms in adults with spastic bilateral cerebral palsy. Dev Med Child Neurol 2012;54:836-42.

11. Ramstad K, Jahnsen R, Lofterod B, Skjeldal OH. Continuous intrathecal baclofen therapy in children with cerebral palsy: when does improvement emerge? Acta Paediatr 2010;99:1661-5.

12. Lundy CT, Doherty GM, Fairhurst CB. Botulinum toxin type A injections can be an effective treatment for pain in children with hip spasms and cerebral palsy. Dev Med Child Neurol 2009;51:705-10.

13. Janzen L, Condliffe E, Toomey C, Kuntze G, Esau S, Brunton $\mathrm{L}$, et al. Influence of regional lean mass on lower-extremity strength in youth with spastic cere- bral palsy. Dev Med Child Neurol 2019;61(S3):111-2.

14. Lai JS, Stucky BD, Thissen D, Varni JW, DeWitt EM, Irwin DE, et al. Development and psychometric properties of the PROMIS pediatric fatigue item banks. Qual Life Res 2013;22:2417-27.

15. Bruni O, Ottaviano S, Guidetti V, Romoli M, Innocenzi M, Cortesi F, et al. The Sleep Disturbance Scale for Children (SDSC): construction and validation of an instrument to evaluate sleep disturbances in childhood and adolescence. J Sleep Res 1996;5:251-61.

16. Kuppuswamy A. The fatigue conundrum. Brain 2017;140:2240-5.

17. Brunton LK. Descriptive report of the impact of fatigue and current management strategies in cerebral palsy. Pediatr Phys Ther 2018;30:135-41.

18. Bolster EA, Balemans AC, de Groot V, Buizer AI, Dallmeijer AJ. Fatigue in children and young adults with physical disabilities: relation with energy demands of walking and physical fitness. Pediatr Phys Ther 2020;32:202-9.

19. Jahnsen R, Villien L, Stanghelle JK, Holm I. Fatigue in adults with cerebral palsy in Norway compared with the general population. Dev Med Child Neurol. 2003;45:296-303.

20. Jahnsen R, Villien L, Egeland T, Stanghelle JK, Holm I. Locomotion skills in adults with cerebral palsy. Clin Rehabil 2004;18:309-16. 